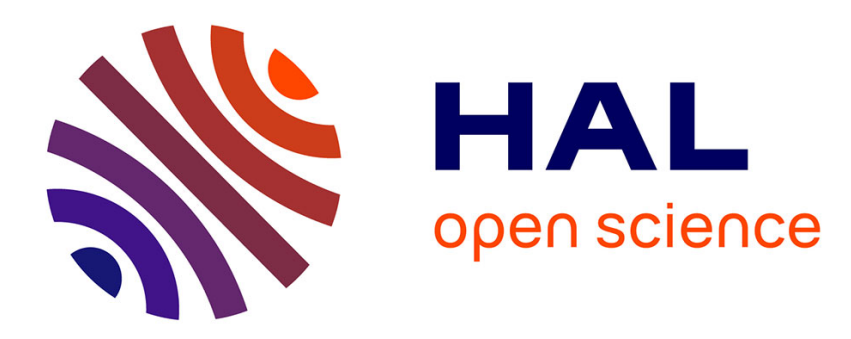

\title{
De la difficulté de rendre des comptes: le cas du risque nucléaire
}

\author{
Emmanuelle Plot, Olivier Vidal
}

\section{To cite this version:}

Emmanuelle Plot, Olivier Vidal. De la difficulté de rendre des comptes: le cas du risque nucléaire.

Gérer et Comprendre. Annales des Mines, 2014, 10.3917/geco.118.0052 . hal-02102911

\section{HAL Id: hal-02102911 \\ https://hal.science/hal-02102911}

Submitted on 10 May 2019

HAL is a multi-disciplinary open access archive for the deposit and dissemination of scientific research documents, whether they are published or not. The documents may come from teaching and research institutions in France or abroad, or from public or private research centers.
L'archive ouverte pluridisciplinaire HAL, est destinée au dépôt et à la diffusion de documents scientifiques de niveau recherche, publiés ou non, émanant des établissements d'enseignement et de recherche français ou étrangers, des laboratoires publics ou privés. 


\section{GÉRER ET COMPRENDRE ; 856}

\section{DE LA DIFFICULTÉ DE RENDRE DES}

\section{COMPTES : LE CAS DU RISQUE}

\section{NUCLÉAIRE}

\begin{tabular}{|l|c|c|}
\hline & Auteur à contacter & Plot \\
\hline Nom & Vidal & Emmanuelle \\
\hline Prénom & Olivier & $\begin{array}{c}\text { PRAG } \\
\text { Titre }\end{array}$ \\
\hline $\begin{array}{c}\text { Maître de conférences } \\
\text { Chercheur au Laboratoire } \\
\text { Interdisciplinaire de Recherche } \\
\text { en Sciences de l'Action, EA } \\
\text { 4603 }\end{array}$ & $\begin{array}{c}\text { Chercheuse au Laboratoire } \\
\text { Interdisciplinaire de Recherche } \\
\text { en Sciences de l'Action, EA } \\
\text { 4603 }\end{array}$ \\
\hline Adresse professionnelle & $\begin{array}{c}\text { CNAM INTEC, 40 rue des } \\
\text { jeuneurs, 75002 Paris }\end{array}$ & $\begin{array}{c}\text { CNAM INTEC, 40 rue des } \\
\text { jeuneurs, 75002 Paris }\end{array}$ \\
\hline
\end{tabular}




\section{DE LA DIFFICULTÉ DE RENDRE DES}

\section{COMPTES : LE CAS DU RISQUE}

\section{NUCLÉAIRE}

\section{Résumé de l'article :}

Un risque extrême est un risque dont la probabilité de survenance est très faible, mais dont les conséquences sont très importantes. Malgré l'existence de trois modes de traitement (provision, passif éventuel et assurance), la traduction du risque dans les états financiers se caractérise par une analyse séquentielle (1) de la probabilité d'occurrence et (2) de la capacité à en estimer les conséquences. Or ce mode d'analyse interdit de rendre compte des risques dont la probabilité de survenance est trop incertaine comme le risque d'accident nucléaire, et ce, indépendamment des montants en jeu. L'étude des rapports de gestion des entreprises concernées par le risque nucléaire montre qu'elles ne compensent pas cette lacune comptable par la diffusion d'informations qualitatives.

\section{Mots clefs :}

Accountability, Provision, Passif éventuel, Risque nucléaire, Probabilité 


\section{THE DIFFICULTY OF BEING}

\section{ACCOUNTABLE : THE CASE OF NUCLEAR}

\section{RISK}

\section{Abstract:}

In spite of the existence of three methods (provisions, contingent liabilities and insurance), the risk in the financial statements is characterized by a sequential analysis of the probability of occurrence and the estimation of the damage which does not allow the nuclear risks to be dealt with. Indeed, every risk whose probability of occurrence is too uncertain does not appear in accounting. Annual reports of companies concerned by the nuclear risk show that they do not compensate for this lack of accountability by disclosing qualitative information.

\section{Keywords:}

Accountability, Provision, Contingent liability, Nuclear risk, Probability 


\section{INTRODUCTION}

La comptabilité est généralement perçue comme un instrument, sinon unique, tout du moins essentiel du processus de communication externe des entreprises. Pourtant, la comptabilité semble bien en peine de remplir sa mission d'information dans de multiples domaines. L'article illustre cette difficulté en présentant un sujet dont la comptabilité peine à rendre compte : les risques extrêmes, et notamment le risque nucléaire.

Une première partie définit la notion de risque en précisant les caractéristiques du risque extrême. Une deuxième partie présente les mécanismes comptables de traduction du risque et plus spécifiquement ceux qui s'appliquent au risque nucléaire. L'incapacité de la comptabilité à traduire de manière satisfaisante ce dernier est présentée dans une troisième partie.

\section{LA NATURE DU RISQUE}

La notion de risque, puis celle de risque nucléaire, méritent d'être clairement définie avant d'aborder les modalités de leur traitement comptable.

\subsection{La notion de risque}

Une définition usuelle du risque ${ }^{1}$ le décrit comme «l'éventualité d'un événement ne dépendant pas exclusivement de la volonté des parties et pouvant causer la perte d'un objet ou tout autre dommage ». Elle foit apparaître deux dimensions : (1) l'éventualité de survenance de l'événement et (2) la conséquence, qui est négative : un dommage.

\footnotetext{
${ }^{1}$ Définition du Petit Robert (dictionnaire de la langue française).
} 
Les deux dimensions (dommage et survenance) peuvent être représentées graphiquement selon deux axes et il est possible de les caractériser en mettant en évidence des niveaux pour chacun des deux axes : l'éventualité de survenance de l'événement peut être incertaine ou certaine et le dommage peut être estimable ou non estimable (voir figure 1).

Figure 1 : Les deux dimensions du risque

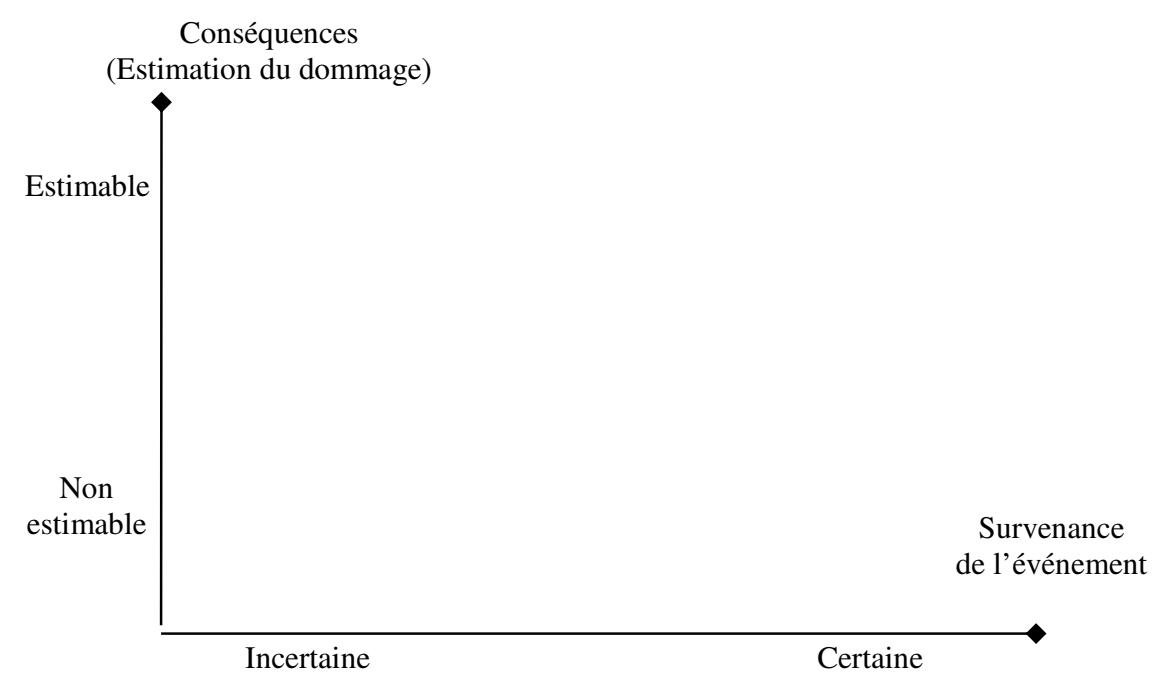

Ainsi, dans la surface créée par les deux axes, quatre zones apparaissent : risque de survenance incertaine avec un dommage non estimable, risque de survenance incertaine avec un dommage estimable, risque de survenance certaine avec un dommage non estimable et risque de survenance certaine avec un dommage estimable.

\subsection{La notion de risque extrême}

Un risque extrême est un risque dont la probabilité de survenance est très faible, mais dont les conséquences sont potentiellement très importantes. Par exemple, le risque d'un accident nucléaire majeur à l'image de celui de Tchernobyl en 1986 ou de Fukushima en 2011 est un risque extrême. L'explosion du site AZF en 2001, les pertes liées aux investissements d'un courtier de la Société Générale en 2008, ou l'accident de la plateforme pétrolière Deepwater Horizon en 2010 sont également des risques extrêmes. 
Ainsi, le risque extrême ne peut être représenté sans ajouter un troisième axe à la figure 1, car la capacité d'estimer les conséquences est indépendante du montant des dommages.

Figure 2 : Un troisième axe pour analyser le risque : l'étendue des dommages

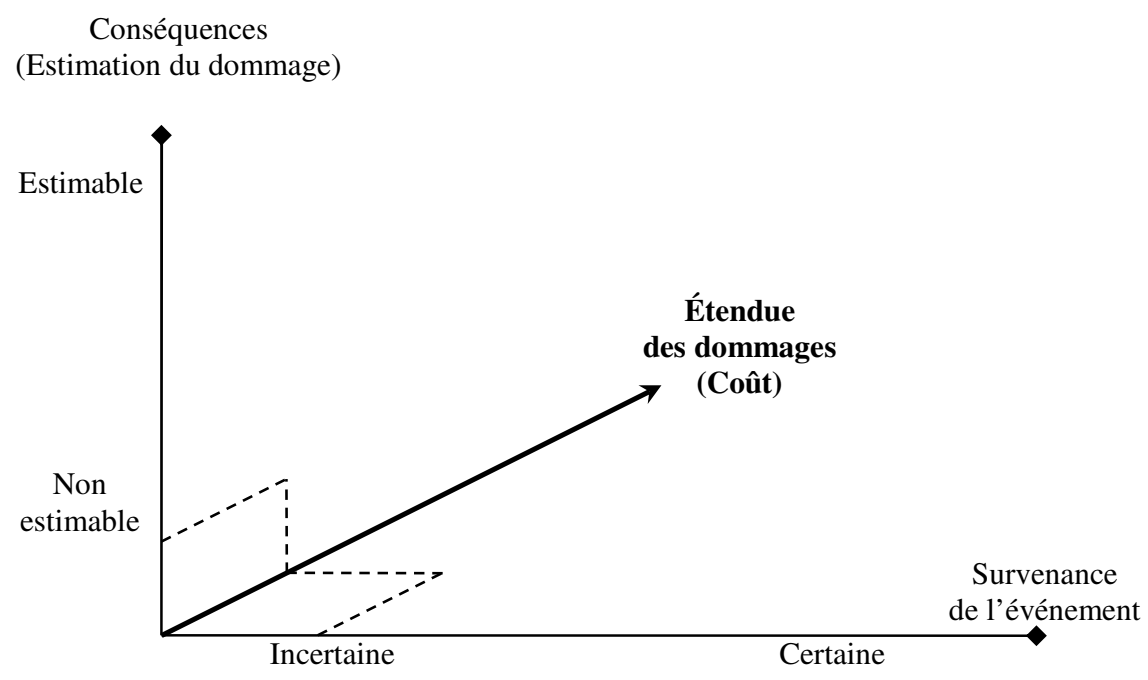

Sur cette nouvelle représentation (figure 2) le risque extrême correspond à une zone qui ne peut être mise en évidence sur la figure 1 en deux dimensions.

Figure 3 : Représentation du risque extrême

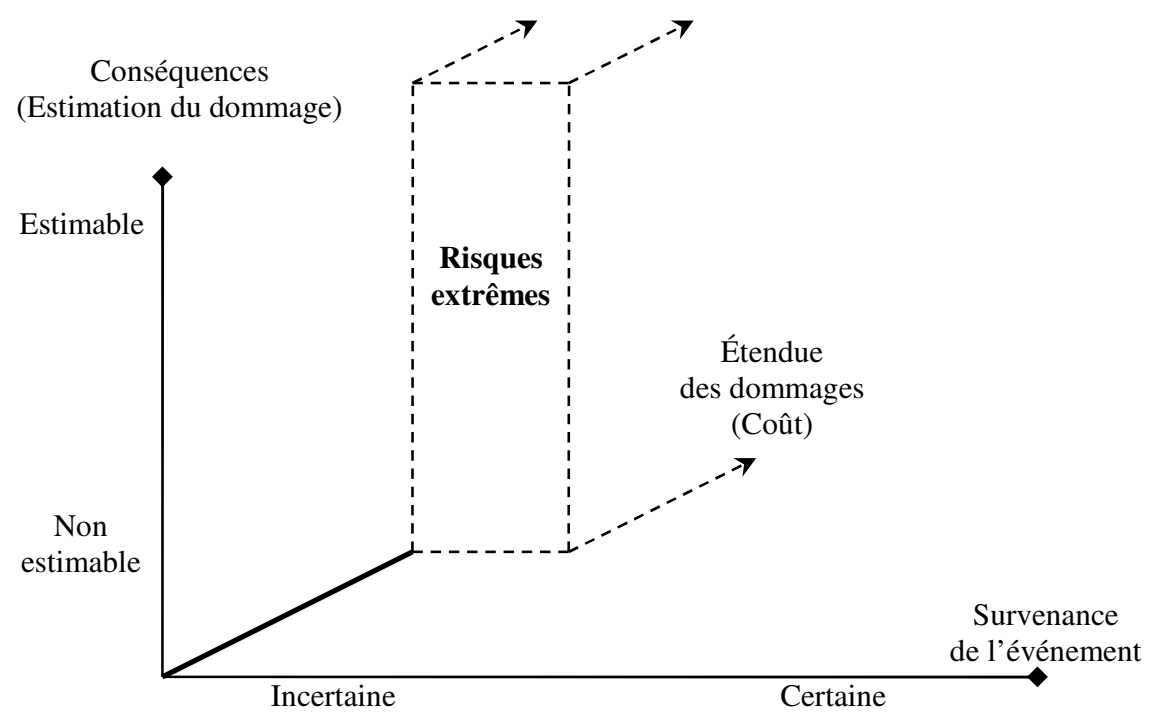

Ce constat permettra d'analyser, dans la deuxième partie de l'article, les limites de l'analyse comptable du risque extrême, et son incapacité à en rendre compte. 


\subsection{La notion de risque nucléaire}

La notion de risque nucléaire n'est pas formellement définie. Toutefois, un point commun apparaît dans toutes les définitions : le risque nucléaire pose la relation entre l'activité industrielle d'une entreprise et la matière radioactive. Il est ainsi indiqué dans le rapport de la cour des comptes (Cour_des_comptes 2005) que le «risque nucléaire résulte de tous les dysfonctionnements susceptibles de mettre des matières radioactives au contact direct ou indirect d'êtres humains ».

La loi relative à la transparence et à la sécurité en matière nucléaire ${ }^{2}$ évoque, pour diminuer le risque nucléaire, deux perspectives complémentaires : d'une part, la mise en place de procédures pour limiter la survenance du risque (sûreté nucléaire ${ }^{3}$ ) et d'autre part, la mise en place de procédures pour protéger les individus et l'environnement des rayonnements ionisants (radioprotection ${ }^{4}$ ). La loi prend donc en compte les deux dimensions observées sur la figure 1 : survenance et conséquences du risque.

Une analyse rapide tend à considérer le risque nucléaire comme un risque extrême. Cependant, cette vision est restrictive et ne recouvre que partiellement la notion de risque nucléaire. Le risque nucléaire doit être considéré sur l'intégralité du cycle de l'activité qui comprend les opérations d'extraction du minerai, de préparation du combustible (enrichissement, fabrication des pastilles...), de traitement des déchets, de stockage (temporaire et permanent) des déchets et de leur retraitement éventuel et du transport entre ces différentes étapes.

\footnotetext{
${ }^{2}$ Loi n ${ }^{\circ 2006-686}$ du 13 juin 2006 relative à la transparence et à la sécurité en matière nucléaire.

3 Définition de la sûreté nucléaire : «ensemble des dispositions techniques et des mesures d'organisation relatives à la conception, à la construction, au fonctionnement, à l'arrêt et au démantèlement des installations nucléaires de base, ainsi qu'au transport des substances radioactives, prises en vue de prévenir les accidents ou d'en limiter les effets » (Loi n²006-686, article 1er).

${ }^{4}$ Définition de la radioprotection : «protection contre les rayonnements ionisants, c'est-à-dire l'ensemble des règles, des procédures et des moyens de prévention et de surveillance visant à empêcher ou à réduire les effets nocifs des rayonnements ionisants produits sur les personnes, directement ou indirectement, y compris par les atteintes portées à l’environnement » (Loi n²006-686, article 1er).
} 
Il ne faut donc pas réduire le risque nucléaire à la seule situation d'accident du type Three Miles Island (1979), Tchernobyl (1986) ou Fukushima (2011). Le risque (extrême) de fusion du cœur n'est qu'un risque nucléaire parmi d'autres. Des risques avec une intensité unitaire moindre et des dommages peu importants peuvent survenir. On parle alors d'incidents. Par ailleurs, le traitement des déchets et le démantèlement présentent une occurrence certaine mais à une date encore indéterminée (Moulin 2003). La diversité des risques nucléaires est représentée graphiquement (figure 4).

Figure 4 : Les deux dimensions du risque nucléaire

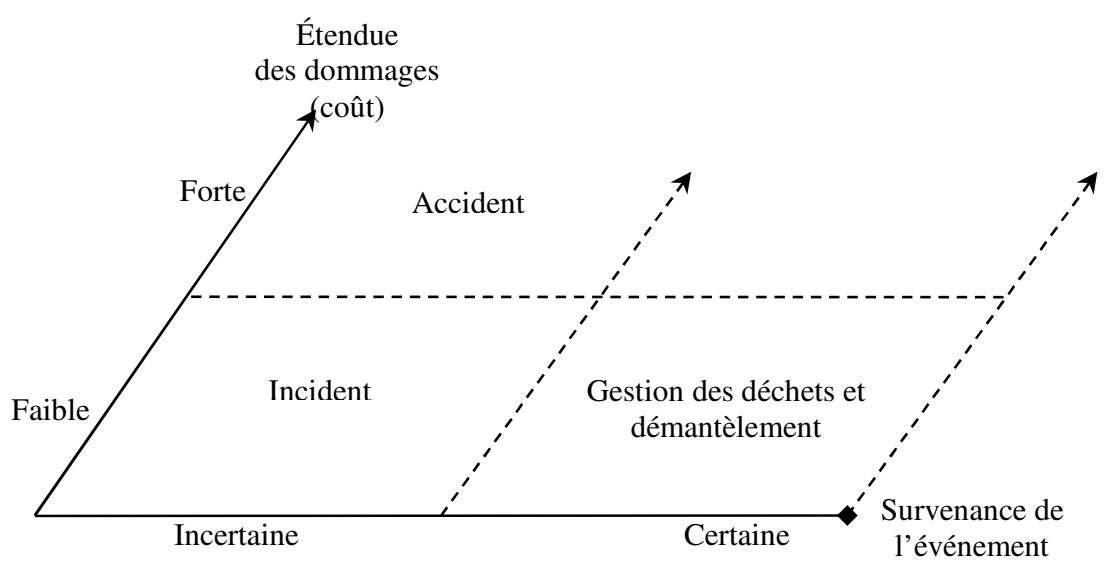

\subsection{Du risque mineur au risque extrême}

Une analyse des incidents nucléaires en France entre juillet 1988 et juin 2008 a été réalisée pour illustrer ce propos. Dans le cadre de l'étude, la base de données du BARPI ${ }^{5}$ a été exploitée. Le moteur de recherche a permis d'identifier 458 événements correspondant à l'activité «production, transport et distribution d'électricité » (code D35.1 dans la base) en France entre le 1 juillet 1988 et le 30 juin 2008. Sur ces 458 événements, seuls 168

\footnotetext{
${ }^{5}$ Depuis 1992, un bureau du Ministère chargé de l'environnement, le Bureau d'Analyse des Risques et Pollutions Industrielles (BARPI) implanté à Lyon, est chargé de rassembler et de diffuser des données sur le retour d'expérience en matière d'accidents technologiques. Une équipe d'ingénieurs et de techniciens assure à cette fin le recueil, l'analyse, la mise en forme des données et enseignements tirés, ainsi que leur enregistrement dans la base A.R.I.A. (Analyse, Recherche et Information sur les Accidents).
} 
concernent des installations nucléaires. Les incidents mineurs ne sont donc pas exceptionnels, puisqu'en moyenne on en dénombre une petite dizaine par an. Ces événements ne sont certes pas exhaustifs mais ils illustrent la diversité des incidents possibles en dehors du risque extrême.

Les tableaux 1 et 2 suivants indiquent que $46 \%$ des événements sont liés à un incendie et $28 \%$ à des défaillances techniques. $58 \%$ des événements n’ont aucune conséquence (économique, sociale ou environnementale).

Tableau 1 : recensement des événements selon leur cause

\begin{tabular}{|l|c|}
\hline Incendies & $77(46 \%)$ \\
\hline Défaillances techniques & $47(28 \%)$ \\
\hline Défaillances humaines & 25 \\
\hline Conditions climatiques & 6 \\
\hline Non précisé & 13 \\
\hline Nombre total des événements sur la période & 168 \\
\hline
\end{tabular}

Tableau 2 : recensement des événements selon leur conséquence

\begin{tabular}{|l|c|}
\hline Aucune & $97(58 \%)$ \\
\hline En termes de radioactivité & 20 \\
\hline Sociales & 10 \\
\hline Environnementales & 6 \\
\hline Financières & 2 \\
\hline Non précisées & 33 \\
\hline Nombre total des événements sur la période & 168 \\
\hline
\end{tabular}

Néanmoins, si les conséquences exceptionnelles des accidents nucléaires justifient une information spécifique, il semble également important de ne pas négliger les risques en apparence mineurs. En effet, la survenance d'un risque majeur suppose une combinaison de plusieurs facteurs d'engrenage. Le risque peut survenir du fait de la succession d'incidents mineurs pris séparément. Ainsi pour le réseau européen SARNET ${ }^{6}$, un grave accident a une infime probabilité d'occurrence parce qu'il suppose une combinaison de plusieurs

\footnotetext{
${ }^{6}$ Réseau SARNET (Severe Accident Research NETwork of excellence) : réseau européen, créé en mars 2004 et composé 200 chercheurs, consacré à la recherche sur les accidents graves de réacteurs. Il permet de fédérer les moyens de recherche disponible en Europe.
} 
défaillances des systèmes de sûreté. Il suppose le cumul de plusieurs événements accidentels et d'une défaillance des systèmes de secours (IRSN 2005). C'est ce qui est constaté lors de l'accident de Fukushima par Kojiro Iaikura, professeur de sismologie à Kyoto: «La catastrophe nucléaire n'a pas été causée par le séisme mais par une série de carences d'origine humaine $»^{7}$.

La préoccupation première est donc d'éviter la survenance d'un incident même banal ou alors de le maitriser très rapidement avant toute autre aggravation. Ce qu'il faut envisager, c'est qu'un «accident banal telle la chute ou le malaise d'un opérateur, la collision ordinaire entre deux véhicules, la rupture ou la fuite sur une vanne ou un joint peut rapidement induire une situation de risque majeur » (Moulin 2003). La capacité à rendre des comptes sur les incidents qui peuvent paraître mineurs peut avoir toute son importance car elle fournit une information importante sur la capacité de l'entreprise à faire face à ces risques, et à les contrôler, donc à limiter le risque d'accident majeur.

Enfin, le risque nucléaire peut avoir des conséquences directes, mais également de graves conséquences indirectes. Dans son document de référence 2007, EDF précise ainsi qu'un accident pourrait avoir des conséquences significatives sur le groupe comme un rejet par l'opinion publique, un durcissement des conditions d'exploitation des centrales, voire la fin de l'énergie nucléaire. Il est remarquable que de telles conséquences indirectes sont possibles même si l'accident n'intervient pas dans une centrale dont l'entreprise est l'exploitant. En mars 2011, dans les quelques jours qui ont suivi l'accident de Fukushima, l'ensemble du secteur nucléaire mondial (composé de 65 groupes internationaux dont les entreprises françaises Areva et EDF) a vu sa capitalisation boursière chuter de près de 100 milliards d'euros. La catastrophe a en effet entraîné l'arrêt ou le ralentissement de nombreux projets dans le monde, et le durcissement des législations.

\footnotetext{
${ }^{7}$ Dans le quotidien Libération du 21 et 22 mai 2011.
} 
Puisque le risque nucléaire comporte des dimensions variées, il est intéressant d'observer comment ces risques sont traduits par la comptabilité. Est-il possible d'informer les parties prenantes sur l'intégralité du risque nucléaire supporté par l'entreprise ?

\section{LE TRAITEMENT COMPTABLE DU RISQUE}

Après une description de la nature de l'information comptable, les modes de traitements des risques par la comptabilité sont décrits. L'analyse de leur mise en œuvre dans le cadre de la traduction des risques nucléaires fait apparaître des lacunes.

\subsection{La nature de l'information comptable}

Il est généralement admis que la comptabilité est un système d'information permettant de fournir des indicateurs utiles à la prise de décision. Malgré un caractère quantitatif très prononcé (la comptabilité s'exprime en unités monétaires), le système d'information comptable ne se résume pas à la production des états financiers. Elle englobe le processus de collecte et de traitement des informations et a de multiples interactions avec l'organisation de l'entreprise.

Par ailleurs, les utilisateurs de l'information comptable sont de natures très diverses. Dans le cadre de la relation d'agence entre dirigeant (agent) et propriétaire (principal), la comptabilité est un instrument utilisé par le principal pour juger les actions de l'agent. Mais le rôle de la comptabilité s'étend au-delà de cette relation: elle sert à de multiples créanciers (banques, fournisseurs...) mais aussi à un ensemble plus large de parties prenantes (État, salariés, analystes financiers, journalistes, collectivités publiques...) qui peuvent être conduits à porter un jugement sur l'entreprise. Cette pluralité conduit le système comptable à produire des informations variées. 


\subsubsection{Deux définitions de la comptabilité}

Une définition restrictive de la comptabilité se limite au processus de production des documents de synthèse (bilan et compte de résultat), centré sur le mécanisme de l'enregistrement en partie double. Le résultat comptable apparaît alors comme un indicateur synthétique de la santé de l'entreprise. Il est complété par des informations sur la structure du patrimoine visant à informer sur la pérennité de l'entreprise, et notamment sa capacité à faire face aux aléas futurs de la vie économique (endettement, fonds de roulement...). Les informations ainsi produites sont financières par nature.

Une acception plus large étend la définition de la comptabilité à un ensemble plus vaste d'informations, parfois qualitatives et peu formalisées, publiées par les entreprises à destination des investisseurs (rapport de gestion, lettre aux actionnaires, sites Internet...). Ces informations sont alors financières par destination, et sont visées par les commissaires aux comptes au même titre que les documents de synthèse.

On retrouve cette distinction entre définition restrictive et étendue de la comptabilité dans l'évolution de la terminologie de la réglementation comptable. Ainsi, les IAS (normes internationales de comptabilité) sont devenues les IFRS (normes internationales de reporting financier). Cette distinction est utile car l'étude des risques extrêmes met en évidence la difficulté de la comptabilité à rendre des comptes, aussi bien à travers les états financiers (conception restrictive de la comptabilité) qu'à travers l'ensemble plus vaste des informations diffusées aux investisseurs (conception étendue de la comptabilité).

\subsubsection{Le caractère prospectif de la comptabilité}

Si le caractère de reddition des événements passés de la comptabilité n'est généralement pas remis en cause, la comptabilité a également vocation (et c'est ce qui la différencie d'une simple comptabilisation des flux de trésorerie) à fournir une information prospective (et 
subjective). Le résultat, tel que le définit Hicks (1939) correspond au montant qui peut être sorti de l'entité (par exemple sous forme de dividende) sans modifier l'entreprise, donc sa capacité à générer des résultats futurs. Cette dimension prospective est traduite dans les normes comptables par le principe de continuité : les actifs sont évalués sous hypothèse que l'entreprise poursuit son activité, ce qui justifie par exemple la valorisation au coût historique des outils de production (plutôt qu'à leur valeur de marché qui serait privilégiée dans une optique liquidative). Elle donne lieu à un second principe, celui de prudence, qui vise à éviter le transfert des risques présents sur les périodes à venir (article 120-3 du PCG).

Dès lors, toute la question est de savoir quand un risque doit être pris en compte. Si le risque est certain (ou quasi certain) et que le montant de la charge encourue est quantifiable, le traitement comptable semble peu discutable. Si le risque est faible, et que le montant de la charge est faible, son traitement comptable peut être négligé car il ne modifiera pas sensiblement l'image de l'entité. Mais comment la comptabilité rend-elle compte des risques faibles de lourdes pertes, c'est-à-dire des risques extrêmes ? C'est la question à laquelle cet article tente de répondre.

\subsection{Le traitement comptable du risque}

Face au risque, la comptabilité propose trois modes de traitement: (1) la provision, (2) l'information en annexe (passif éventuel), et (3) l'assurance.

\subsubsection{Les provisions}

La réglementation française (CRC 2000) définit une provision comme un passif dont l'échéance et le montant ne sont pas fixés de façon précise. Si l'événement est certain, et que son échéance et son montant sont fixés de façon précise, alors l'entité enregistre une dette et non une provision. La norme IAS 37 précise qu'une provision existe lorsque (1) il existe une 
obligation actuelle à l'égard d'un tiers et résultant d'un événement passé (2) qui provoquera de manière certaine ou probable une sortie de ressource («plus probable qu'improbable ») (3) mais que son montant n'est pas fixé de manière fiable.

Autrement dit, les deux axes (survenance et conséquences) sont ici décomposés. L'axe « survenance » est divisé selon deux dimensions : l'obligation et la sortie de ressource. Si la sortie de ressource (la nature dommageable de l'événement) peut n'être que probable, l'obligation doit être actuelle. L'entité ne doit pas faire des paris sur l'avenir. Par exemple, un litige en cours génère une obligation actuelle, même si le résultat du procès demeure incertain. Une provision peut alors se justifier. Par contre, l'entreprise ne doit pas provisionner pour des procès éventuels qui n'ont encore aucun début d'existence.

Le deuxième axe «conséquences » n'est, pour sa part, analysé que sous l'angle de la fiabilité de son estimation. La lecture des normes nationales comme internationales conduit donc à constater que le comptable ne distingue pas les risques en fonction de l'ampleur de leurs éventuelles conséquences (voir figure 5). Les normes IFRS en vigueur depuis 2005 pour les comptes consolidés des sociétés cotées n'ont apporté aucune modification substantielle.

Figure 5 : Dettes et provisions

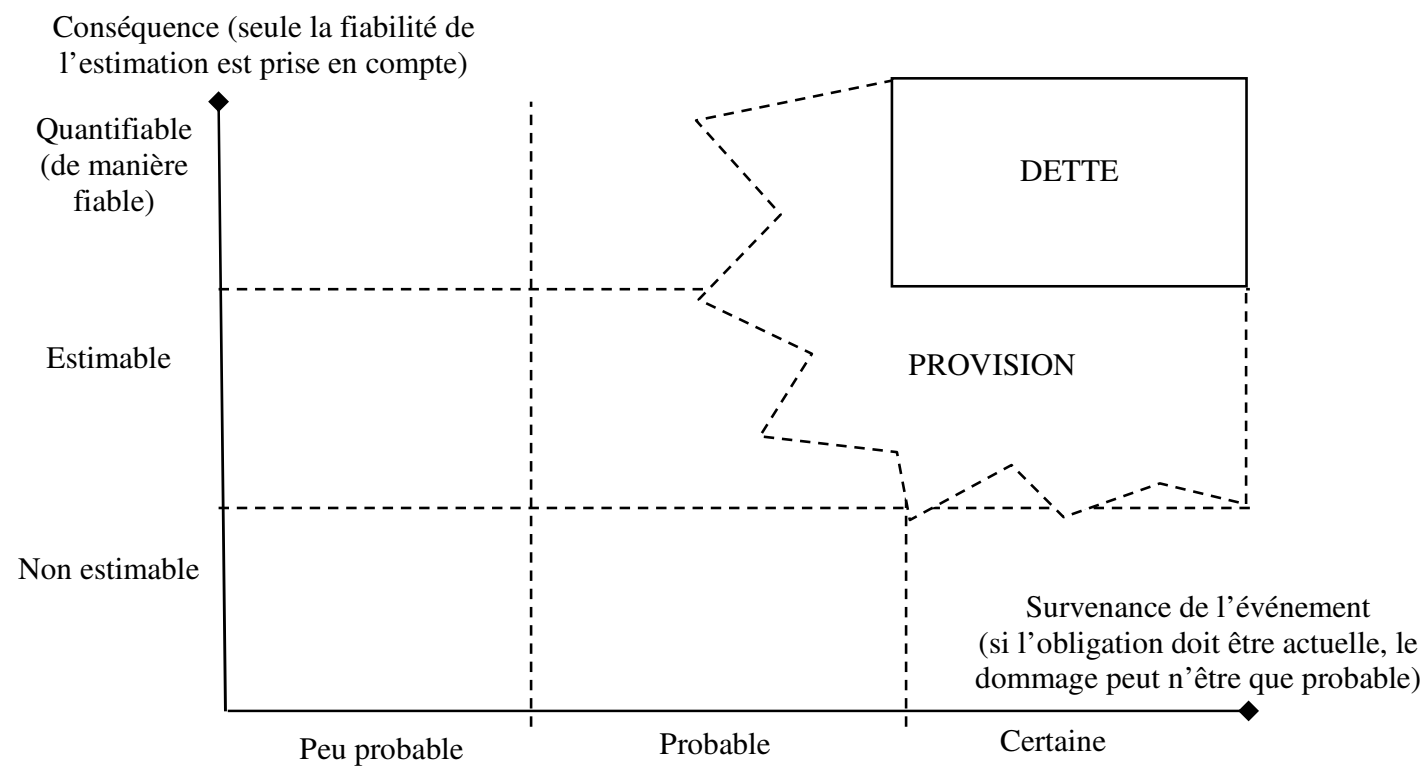


Il demeure une certaine part d'imprécision pour définir les frontières (en pointillé sur la figure 5) des provisions (Dick et Eberhartinger 2002). Cette marge s'apprécie pleinement dans l'expression «plus probable qu'improbable» de l'IAS 37. Comment définir le passage entre une situation probable et une situation improbable ? Il faudrait pour cela définir clairement les probabilités de survenance mais de telles précisions dans les estimations ne sont généralement pas possibles.

\subsubsection{Les passifs éventuels}

Le normalisateur français (CRC 2000) a repris pour l'essentiel la définition de l'IAS 37 : «Un passif éventuel est : (1) soit une obligation potentielle de l'entité à l'égard d'un tiers résultant d'événements dont l'existence ne sera confirmée que par la survenance, ou non, d'un ou plusieurs événements futurs incertains qui ne sont pas totalement sous le contrôle de l'entité ; (2) soit une obligation de l'entité à l'égard d'un tiers dont il n'est pas probable ou certain qu'elle provoquera une sortie de ressources sans contrepartie au moins équivalente attendue de celui-ci ».

Par ailleurs, le CRC précise que « un passif n'est pas comptabilisé dans les cas exceptionnels où le montant de l'obligation ne peut être évalué avec une fiabilité suffisante » (voir figure 6). 
Figure 6 : Passifs éventuels et information en annexe

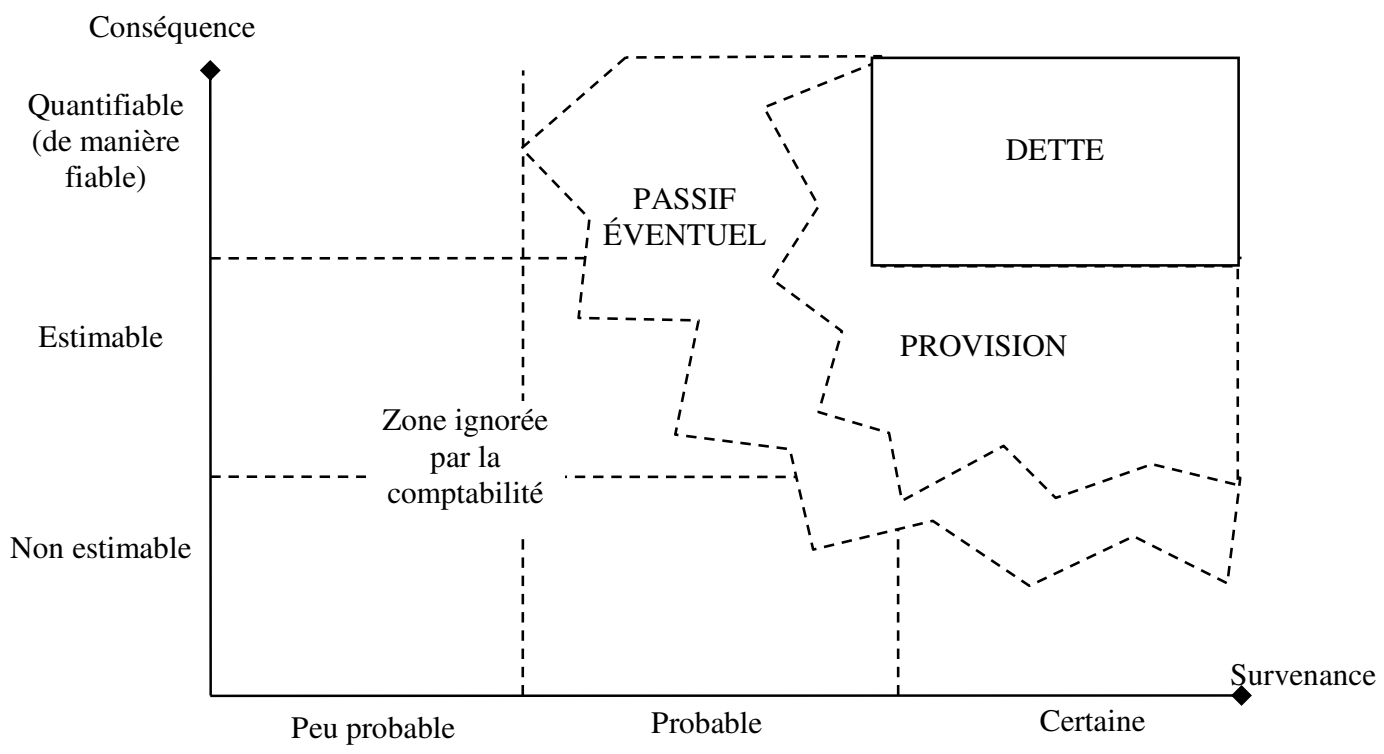

Les passifs éventuels ne donnent pas lieu à comptabilisation (au bilan ou compte de résultat), mais à une information en annexe $\mathrm{e}^{8}$ dans la mesure où leur importance est significative. Ils entrent donc dans la catégorie des informations financières par destination.

Mais renoncer à quantifier l'impact en valeur monétaire de ces évènements conduit à ne pas intégrer ces risques dans les charges de l'entreprise. Une information en annexe peut alors être considérée comme un renoncement, un palliatif. La conséquence très concrète de ce renoncement se matérialise dans le prix de vente du produit (le $\mathrm{kWh}$ d'origine nucléaire par exemple) qui peut être sous-estimé car il n'intègre pas la totalité des coûts (actuels et futurs) liés à sa production ${ }^{9}$.

\footnotetext{
${ }^{8}$ D'après la norme IAS 37, l'entreprise doit fournir en annexe, comme information relative au passif éventuel, une brève description de ce passif éventuel et dans la mesure du possible une estimation de son effet financier, une indication des incertitudes relatives au montant ou à l'échéance de toute sortie et la possibilité de tout remboursement.

${ }^{9}$ Remarquons que, même si le coût de l'accident potentiel n'est pas pris en compte, les coûts générés pour maintenir le risque à un certain seuil acceptable (coûts de contrôle, de sécurité...) sont eux pris en compte. Le coût de la gestion de la sécurité nucléaire n'est pas extra-comptable. Ceci étant dit, le niveau de risque jugé acceptable n'est pas issu d'une norme comptable. Ainsi, d'un pays à l'autre, si les ingénieurs n'ont pas la même perception du niveau « acceptable » du risque, les coûts liés à la sécurité seront différents.
} 


\subsubsection{L'assurance}

Le troisième traitement proposé est celui de la comptabilisation d'une prime d'assurance lorsque le risque est couvert. Dans cette situation, il est remarquable de noter qu'aucune analyse de la nature du risque n'est nécessaire. Que la survenance du risque soit probable ou pas, que son estimation soit quantifiable ou non, ces problèmes sont externalisés vers l'organisme d'assurance qui facture le prix de son service. Pour l'entreprise, ces primes sont enregistrées en charges. Le coût de l'assurance est donc comptabilisé, et les conséquences éventuelles ne sont plus supportées par la seule entreprise. Elles sont statistiquement réparties sur un grand nombre d'intervenants.

La traduction comptable du risque couvert par une assurance n'est cependant pas stricto sensu un traitement comptable du risque puisqu'elle contourne le problème de l'analyse des risques extrêmes. Cette solution technique n'est satisfaisante que si le risque est couvert en totalité. Or ce n'est généralement pas le cas. L'externalisation de l'analyse du risque se limite à la partie couverte par l'organisme extérieur alors que les assurances prévoient généralement des plafonds de couverture.

\subsection{Le traitement comptable du risque nucléaire}

La traduction comptable du risque nucléaire est examinée au travers des trois modes présentés précédemment. Il convient de distinguer plusieurs situations car le risque nucléaire n'est pas une notion unidimensionnelle.

\subsubsection{Les provisions}

L'enregistrement en comptabilité d'une provision nécessite la réunion de trois conditions déjà présentées. Deux situations répondent à cette exigence : le démantèlement des installations nucléaires et la gestion des déchets radioactifs. 
Ces deux opérations provoquent un risque nucléaire car la matière radioactive est au contact des individus et de l'environnement. L'obligation est actuelle : les exploitants d'installations nucléaires doivent démanteler les sites nucléaires et gérer les déchets. Dans l'avis du CNC (2005) relatif au démantèlement, il est précisé que la provision doit être constatée en totalité dès la réalisation de l'installation. L'obligation existe du fait même de la construction ou de la mise en service, car la sortie de ressources est inéluctable, quel que soit le niveau d'exploitation de l'installation ou du site concerné. Enfin, les montants sont estimables avec une relative fiabilité. Les trois conditions sont donc réunies.

Par exemple, le Groupe EDF dans son rapport annuel de 2012 indique deux provisions pour risques et charges nucléaires : un montant de provisions pour aval du cycle nucléaire (gestion des déchets) de 19525 millions d'euros et un montant de provisions pour déconstruction et pour derniers cœurs de 20979 millions d'euros. Aucune autre provision ne concerne le risque nucléaire.

\subsubsection{Les passifs éventuels}

L'avis du CNC relatif au démantèlement mentionné ci-dessus évoque rapidement l'existence de passifs éventuels en indiquant qu' «aucune provision ne sera comptabilisée, dans les cas exceptionnels où le montant de l'obligation ne peut être évalué avec une fiabilité suffisante. Dans ce cas l'obligation constitue un passif éventuel ». Mais aucun exemple n'est cité pour illustrer l'une ou l'autre de ces situations.

On s'attendrait donc à trouver, dans les annexes des états financiers, un certain nombre d'informations concernant les risques nucléaires. L'étude menée sur les états financiers du groupe EDF est très décevante à ce sujet. En effet, aucune information n'y figure. Les incidents ou accidents ont une probabilité de survenance jugée trop incertaine pour justifier l'existence de passifs éventuels: les obligations sont potentielles et les dommages 
difficilement estimables. Il en découle que la notion de passif éventuel, telle qu'elle est appliquée par les entreprises, ne permet pas la prise en compte du risque nucléaire.

Pourtant, cela ne signifie pas qu'il n'y a pas de risque. Chaque année, des incidents nucléaires surviennent (168 incidents sont survenus en France entre 1988 et 2008). Le risque n'est donc pas nul, mais la comptabilité n'est pas à même d'en rendre compte.

\subsubsection{L'assurance}

Enfin, les états financiers sont affectés par les risques nucléaires d'occurrence incertaine qui sont couverts par une assurance. La prime d'assurance est une charge d'exploitation qui varie selon le type d'installation nucléaire et les règles en vigueur dans chaque pays. Jusqu'en 2011, le Groupe EDF, dans ses rapports annuels, ne donnait aucune explication sur son système d'assurance en cas d'incident ou d'accident nucléaire. Il faut attendre le rapport 2011 pour trouver une information sur l'existence d'une convention. En 2012, EDF indique être couvert à hauteur de 91,5 millions d'euros.

Malgré ces informations lacunaires, un régime général existe pour les accidents graves dans le secteur nucléaire. Ce régime de la responsabilité civile nucléaire pour les accidents graves présente un caractère particulier car il concerne uniquement les événements ayant une très faible probabilité d'occurrence mais dont les conséquences pourraient être considérables (Bertel et Naudet 2004), c'est-à-dire les risques extrêmes.

Les conventions de Paris (1960), de Bruxelles (1963) et de Vienne (1963) traitent de la responsabilité de l'exploitant nucléaire et précisent ses obligations en termes de garanties financières. L'objectif de ces conventions était de maximiser les montants mis à disposition des victimes en cas d'accident majeur et d'inciter les exploitants à réduire le risque d'accident (Schmitt et Spaeter 2007). Mais elles sont aujourd'hui considérées comme désuètes (Berkowitz 2013). 
Les exploitants nucléaires sont responsables des conséquences financières à hauteur d'une première tranche $\left(700\right.$ millions d'euros $\left.{ }^{10}\right)$. Les indemnisations complémentaires relèvent dans un premier temps du pays dans lequel l'accident a lieu et dans un deuxième temps de l'ensemble des pays signataires. Au-delà, c'est l'exploitant qui est responsable, mais les législations nationales peuvent limiter cette responsabilité. Ainsi, en Allemagne, la responsabilité civile de l'exploitant nucléaire est illimitée alors qu'en France, elle est limitée à la seule première tranche de 700 millions d'euros. L'État français garantit 500 millions d'euros supplémentaires, et l'ensemble des autres États signataires une tranche supplémentaire de 300 millions d'euros. Au japon, un système d'assurance, le «pool nucléaire » permet de faire face jusqu'à 61 milliards de Yens (530 millions d'euros). Au-delà, c'est l'État qui prend le relais.

Chez EDF, les exigences de sûreté imposent une probabilité de survenance d'un accident de fusion de cœur d'un réacteur de type REP soit de l'ordre de 1 sur 100000 par annéeréacteur ${ }^{11}$. La sortie de ressource est donc «moins probable que probable », et pourtant, les conséquences financières d'un accident grave de réacteur sont évaluées, selon les hypothèses de calcul du projet ExternE, à 17 milliards $^{12}$ d'euros (Bertel et Naudet 2004). Les 700 millions d'euros exigés par le régime de la responsabilité civile nucléaire sont donc largement dépassés.

Il convient donc de conserver un regard critique quant à ce système d'assurance et le Groupe EDF lui-même indique dans son document de référence 2007 que «le Groupe ne peut pas

\footnotetext{
${ }^{10}$ Étrangement, le rapport de gestion d'EDF ne parle pas de cette convention.

${ }^{11}$ En 2012, près de 400 réacteurs nucléaires fonctionnent dans vingt-huit pays différents dans le monde (dont une soixantaine en France). Le premier réacteur a été construit aux États-Unis en 1942. Si l'on considère qu'en près d'un demi siècle, le nombre de réacteur-année est de l'ordre de $(400 * 60) / 2$, soit 12000 , et que trois accidents majeurs ont eu lieu, il est possible de penser que la probabilité réelle d'accident est très supérieure à la norme technique (de l'ordre de 1 sur 4 000).

${ }^{12}$ L'espérance mathématique du risque nucléaire extrême peut donc être évaluée à $17 \mathrm{Mds} / 100000=170000$ euros. Mais quelle signification attribuer à un tel chiffre au regard des montants réels en cas de survenance de l'accident?
} 
garantir que, dans les pays où il est exploitant nucléaire, les plafonds de responsabilité fixés par la Loi ne seront pas augmentés ou supprimés » (p. 24). De plus, il semble raisonnable de penser qu'un accident nucléaire a des incidences allant bien au-delà de celles couvertes par le régime de la responsabilité civile de l'exploitant, notamment du fait des conséquences indirectes de l'accident. Par exemple, deux mois après la catastrophe nucléaire de Fukushima, le groupe TEPCO, exploitant de la centrale, annonçait une perte de 11 milliards d'euros n'incluant pas les dédommagements à verser aux dizaines de milliers de familles et entreprises touchées par la catastrophe, et le départ de son PDG, démis de ses fonctions.

En définitive, l'intérêt d'étudier les risques nucléaires réside dans le fait qu'ils couvrent l'ensemble des situations de risque précédemment décrites. Leur traitement comptable illustre les limites évoquées dans la première partie: seuls les risques dont la survenance et l'échéance sont quasi certaines sont traduits sous forme de provision. Les risques assurés le sont pour des montants importants, mais qui demeurent en deçà du coût réel d'une catastrophe majeure. Enfin, les informations en annexe sont absentes et ne traduisent pas l'existence de risques qui ne sont pourtant pas imaginaires. En conclusion, la comptabilité ignore largement les risques nucléaires de manière générale, et notamment les risques nucléaires extrêmes.

\section{L'IMPOSSIBILITÉ DE RENDRE COMPTE}

La comptabilité ignore les risques peu probables ou difficilement estimables. Pourtant, parmi ces risques aux contours flous, certains peuvent concerner des événements dont les conséquences financières, sociales, humaines, écologiques peuvent être considérables. Les investisseurs (comme ceux du groupe TEPCO qui exploite la centrale de Fukushima) ne sontils pas légitimement intéressés par une information sur de tels risques ? De même, si le risque encouru avant 2008 par la Société Générale de faire une perte de 5 milliards d'euros était 
extrêmement faible, des scénarios catastrophes sont étudiés dans les banques qui envisagent de tels événements. Dès lors que l'événement est envisageable par la direction, la comptabilité ne devrait-elle pas en rendre compte?

Pourquoi la comptabilité ne traduit-elle pas les risques extrêmes ? Cette question sera abordée sous trois angles : celui du rapport de la comptabilité aux probabilités, celui de la focalisation de la comptabilité sur les échéances, et celui des enjeux sociaux de la comptabilité.

\subsection{Provisions et probabilité}

La définition du risque a mis en évidence deux dimensions : sa survenance (probabilité) et ses conséquences (montant du dommage). Une estimation mathématique du risque tendrait à multiplier les deux termes selon une équation du type : $\mathrm{E}(\mathrm{R})=\mathrm{P} \times \mathrm{M}$

Avec $\mathrm{E}(\mathrm{R})=$ Espérance du risque (ou coût moyen du dommage encouru) $; \mathrm{P}=$ Probabilité de survenance $; \mathrm{M}=$ Montant estimé du dommage.

Par exemple, si une entreprise a une probabilité annuelle de $1 \%$ de faire 1 milliard de pertes, son espérance de perte annuelle (risque moyen) peut être évaluée à 10 millions. Si une seconde entreprise a une probabilité annuelle de $99 \%$ de faire 10 millions de perte, son risque moyen est sensiblement identique. Pourtant, dans le premier cas, le résultat comptable n'est pas modifié, alors que dans le second cas, le résultat est provisionné de 10 millions (pour un risque moyen de 9,9 millions).

Mathématiquement, il apparaît que le coût moyen du risque augmente de manière progressive lorsque la probabilité augmente, ou lorsque le coût des conséquences encourues augmente. Or pour la comptabilité, le coût du risque est binaire ; seules les probabilités proches ou égales à 1 sont prises en compte. Il y a une asymétrie dans le traitement comptable du risque dû à une hiérarchisation des deux dimensions probabilité et coût. Le processus de raisonnement peut être illustré sous forme d'arbre de décision (voir figure 7). 
Figure 7 : Processus d'analyse comptable du risque

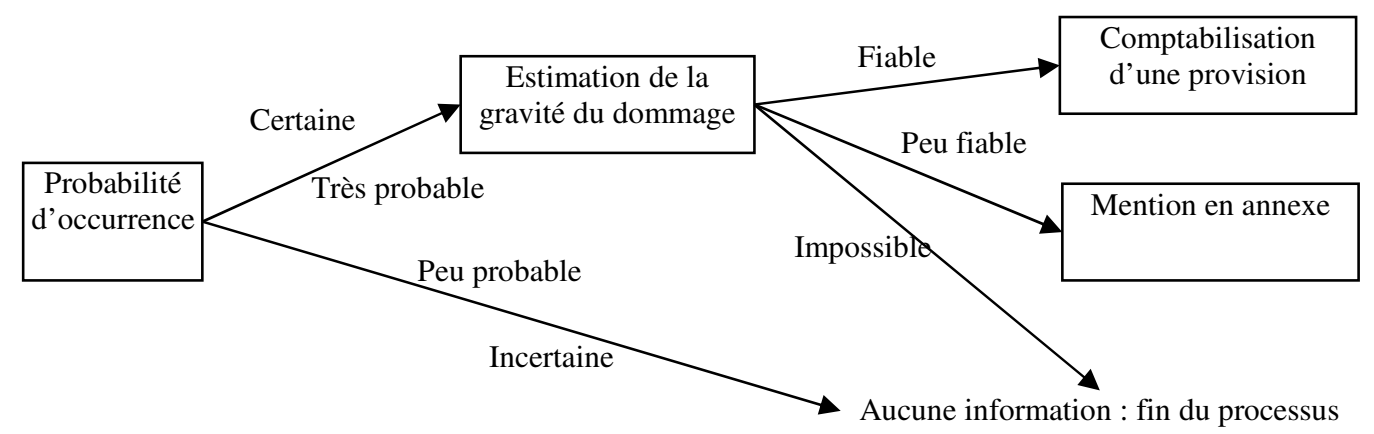

Autrement dit, la comptabilité analyse la nature de l'événement avant d'en estimer son coût. Le montant des conséquences n'est pas pris en compte dans le raisonnement. De surcroît, il n'intervient que si (1) la probabilité de survenance est suffisamment proche de 1, et (2) s'il est estimable de manière suffisamment fiable. Ce constat conduit à la question : pourquoi la comptabilité analyse-t-elle le risque de manière séquentielle ?

Si le processus d'analyse séquentielle est pur d'un point de vue logique, il manque de pragmatisme puisqu'une catastrophe importante est analysée de la même manière qu'un événement mineur. Cette approche se fait au détriment de l'image fidèle. Cette approche, où prudence l'emporte sur pragmatisme, est opposée à celle des ingénieurs. Ainsi, Moulin (2003) écrit que «pour apprécier la gravité d'un risque, c'est la gravité potentielle des conséquences de l'accident qui doit être la référence et non pas le taux d'occurrence probable car un risque à probabilité d'occurrence faible peut être de grande ampleur par ses conséquences». L'accident récent de Fukushima rappelle à quel point cette assertion est vraie.

L'approche séquentielle est donc avant tout privilégiée par le comptable car elle lui fournit un cadre d'analyse permettant de répondre à la principale question qui le préoccupe et qui se réduit à une question binaire : faut-il ou non inscrire quelque chose dans les comptes ? En évacuant la question de l'importance des conséquences, ce traitement ignore les risques extrêmes. 
Il serait donc intéressant de faire apparaître une troisième dimension dans l'analyse du risque qui, pour être représentée graphiquement, nécessite un troisième axe (voir figure 2). On comprend dès lors que le risque extrême est ignoré par la comptabilité qui ne rend compte que de la « surface » du « cube des risques » représenté dans la figure 8.

Figure 8 : Prise en compte de l'importance du dommage

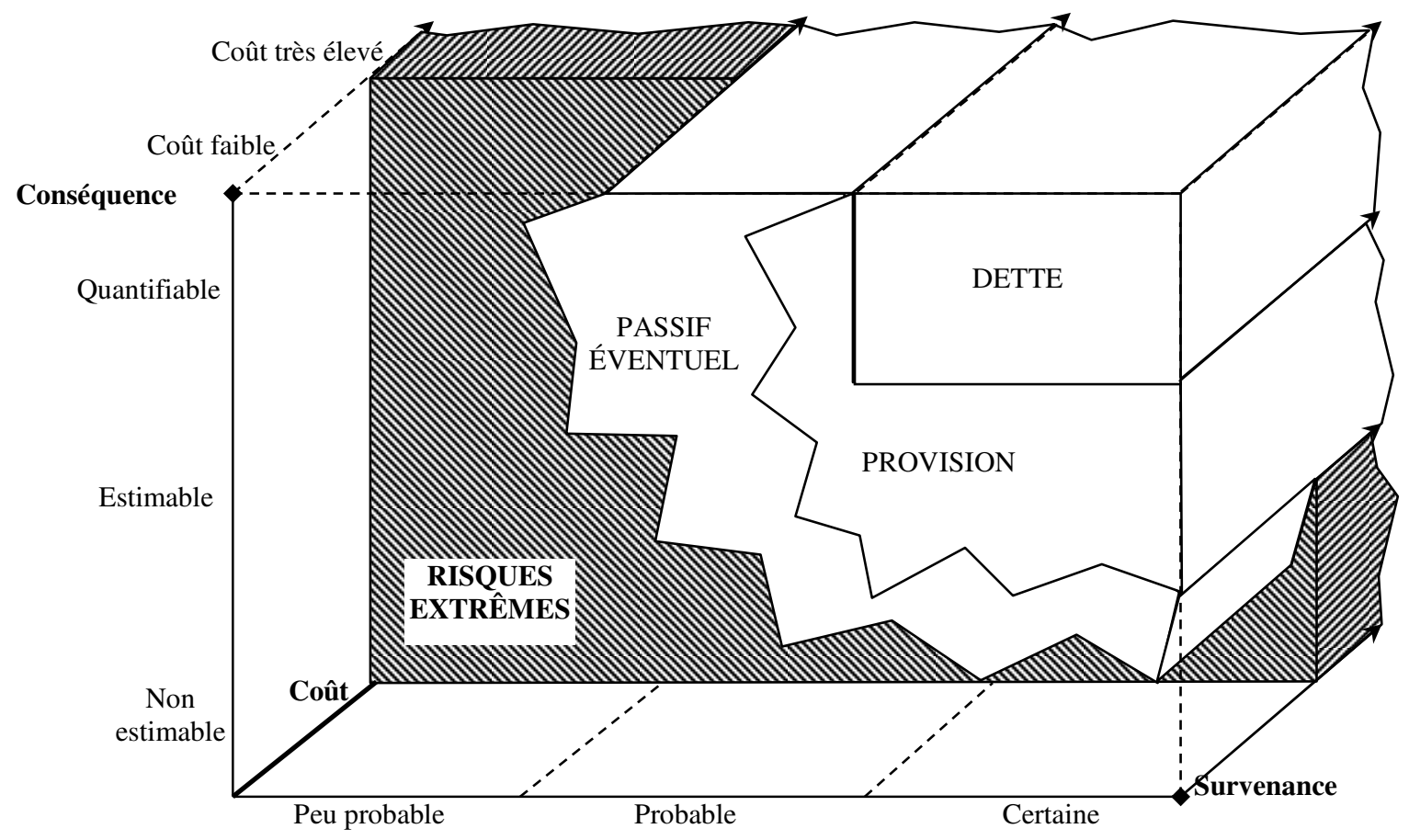

Si la comptabilité évite les calculs de probabilités, c'est en partie pour des raisons techniques (mathématiques). Les modèles de probabilité ont longtemps été bannis des états financiers. S'ils commencent à être envisagés (comme dans le cas de la norme IFRS 2 sur la comptabilisation des stocks-options qui depuis 2005 s'appuie sur le modèle mathématique de Black et Scholes), ils doivent cependant avoir fait leurs preuves avant d'être reconnus et intégrés dans un système d'informations qui est fondé sur la prudence et qui cherche à limiter les arbitraires. 
Mais au-delà des raisons techniques, c'est avant tout le processus d'analyse du risque qui, en se voulant logique, perd en pragmatisme ${ }^{13}$. La hiérarchisation de l'analyse en fonction de la nature du risque, au détriment du coût encouru nuit à l'objectif d'image fidèle, notamment en cas de risques extrêmes.

\subsection{Provisions et échéances}

Pour l'ingénieur, l'analyse du risque doit le conduire à mettre en œuvre une solution pour maintenir ce risque à un niveau acceptable. Pour le comptable, l'analyse du risque le conduit à déterminer s'il doit enregistrer ou non l'événement, c'est-à-dire informer les parties prenantes. C'est une question binaire, qui n'a pas vocation à influencer (directement) le risque. Le rôle de l'ingénieur est d'agir sur le risque (rôle actif), celui du comptable se limite à rendre compte (rôle passif en apparence).

Dès lors, on peut comprendre que l'ingénieur oriente son analyse sur les conséquences alors que le comptable accorde plus d'importance à la question de l'échéance. Quand doit-il informer du risque ? À partir de quand ne peut-il plus l'ignorer?

L'échéance occupe un rôle clef dans le questionnement et explique l'importance accordée à la probabilité de survenance au détriment du coût des conséquences. Les provisions pour engagement de retraite illustrent ce propos. L'événement (départ à la retraite d'un salarié) n'est pas certain, mais l'échéance est connue si le salarié demeure dans l'entreprise jusqu'au terme de son contrat de travail. Dès lors, s'il est possible d'estimer (même approximativement) les coûts, il devient possible de constituer une provision étalée dans le temps. Si l'échéance était inconnue, il serait impossible de constituer une provision progressive.

\footnotetext{
${ }^{13}$ Il est utile de préciser qu'une entreprise qui exploite une seule centrale est confrontée à un risque extrême faible. Mais une entreprise qui exploite la soixantaine de centrales nucléaires situées en France est confrontée à un risque soixante fois plus élevé. L'analyse séquentielle ignore totalement cette information.
} 
Force est de constater que l'idée même de provisionner un risque peu probable semble fragile. Dans l'exemple précédent (voir paragraphe 3.1), quel sens y aurait-il à ce que l'entreprise qui a $1 \%$ de chance de faire un milliard de pertes provisionne un montant de 10 millions ? Soit l'événement ne survient jamais (ce qui est le plus probable) et la provision est inutile, soit l'événement survient et la provision de 10 millions qui sous-estime largement les conséquences du risque est inutile également.

En conclusion, dans le cas des risques nucléaires, il n'y a pas d'échéance. Le comptable, même s'il est informé de l'existence d'un risque potentiellement important, ne sait pas quand enregistrer l'information. Son mode d'analyse est le reflet du rôle d'informateur neutre qu'il endosse. C'est pourquoi il accorde tant d'importance à l'échéance, quitte à négliger l'importance des conséquences du risque.

Pourtant, informer c'est agir indirectement sur le risque puisqu'en informant, la comptabilité donne la possibilité aux investisseurs (et plus généralement aux parties prenantes) de modifier leur comportement et donc d'influencer les décisions des dirigeants. La distinction entre l'analyse «active » du risque par l'ingénieur, et l'analyse «passive » du comptable n'est pas une distinction inévitable. Il est le résultat d'un partage des rôles accepté par les différents acteurs économiques. Dès lors, une lecture sociopolitique peut-elle expliquer le traitement comptable du risque?

\subsection{Provisions et enjeux sociaux}

La réflexion menée jusqu'alors s'apparente à une démarche normative, visant à décortiquer le mode d'analyse comptable du risque afin de justifier les règles mises en œuvre, et permettant de définir ce qui devrait être fait (ou ce qui devrait changer) face à un événement (Colasse 1999). Le principe de prudence justifie ainsi l'enregistrement de provisions et l'analyse du risque hiérarchisant survenance et conséquences de l'événement conduit à déterminer quand 
une provision doit ou ne doit pas être constatée. C'est une explication normative qui décrit et explique ce qui doit être fait.

Cette approche peut être complétée par une réflexion sur les enjeux sociologiques et politiques du traitement du risque par la comptabilité : Pourquoi l'analyse du risque par les comptables, qui conduit à négliger les risques extrêmes, s'est imposée jusqu'alors et fait consensus malgré ses limites?

Une approche sociologique de la comptabilité justifie l'existence d'une norme dans un contexte social. L'outil comptable est un enjeu au centre de conflits d'intérêts puisqu'il permet aux acteurs d'évaluer les actions d'autres acteurs. Les règles comptables sont donc partagées entre deux objectifs contradictoires : (1) présenter une image fidèle de la réalité économique et (2) garantir la neutralité et la fiabilité de l'information. Le premier objectif nécessite une marge d'appréciation subjective des événements alors que le second nécessite neutralité et objectivité. Face à ce dilemme, le système comptable est le fruit de consensus. Il cherche à produire des règles qui évitent au maximum les choix pour se protéger de toute influence. Mais il est obligé de créer des marges d'appréciations.

Dans ce contexte, comment interpréter les règles de prise en compte des provisions? Pour les dirigeants, les provisions sont une source d'interprétation importante. Elles peuvent donc être un instrument de gestion du résultat en leur faveur. Les propriétaires peuvent alors se méfier de trop de liberté accordée à leurs agents. Le comptable est au cœur de ce conflit d'intérêt : pour se défendre et trouver sa place entre les intérêts divergents, il va tenter d'établir des règles qui limitent les choix, les interprétations.

Pour les propriétaires, les provisions interdisent, par prudence, les distributions excessives et reportent vers le futur les résultats. Les provisions se justifient, soit dans une optique de valorisation à long terme, soit comme instrument de gestion du résultat au détriment des tiers, 
notamment des créanciers ou de l'État. Les propriétaires ont donc un rapport ambigu face aux provisions. S'ils peuvent en profiter ponctuellement, ils sont non seulement dépendants de la bonne foi de leurs agents (les dirigeants), mais cela peut s'opposer à une valorisation de leur patrimoine à court terme qui est pourtant souhaitable en cas de revente de parts. Au total, les propriétaires n'ont pas intérêt a priori et de manière générale à favoriser les provisions.

Pour les créanciers, l'État et les salariés, les provisions diminuent les distributions de résultat et garantissent à long terme la solvabilité de l'entreprise. Mais en contrepartie, les provisions donnent aux entreprises un pouvoir d'appréciation conduisant à des opportunités de manipulation qui peuvent se faire à leur détriment. Contrairement aux propriétaires, les tiers ont intérêt à favoriser les provisions. Mais ils ont également intérêt à les limiter à des situations contrôlables, c'est-à-dire à des situations où le pouvoir discrétionnaire des dirigeants est limité. Sur ce dernier point, l'intérêt des tiers rejoint l'intérêt des propriétaires. En définitive, le consensus conduit à exclure les provisions dans les cas où les événements sont seulement « probables ».

$\mathrm{Au}$ total, il apparaît que les règles concernant les provisions sont un enjeu sociopolitique important puisque les différentes parties prenantes ont des intérêts divergents. Les acteurs les plus favorables à des règles élargissant la constitution de provisions sont les dirigeants (par opportunisme) et les investisseurs extérieurs (par prudence). Dans une situation potentiellement conflictuelle, le consensus est difficile à trouver et la dimension de contrôlabilité s'impose naturellement comme un élément primordial. Cette analyse justifie ainsi le traitement séquentiel des risques (figure 7) et l'ignorance des risques extrêmes.

En conclusion, s'il semble difficile de traduire les risques extrêmes en comptabilité, c'est sans doute autant pour des raisons techniques (difficultés d'utiliser des calculs probabilistiques, problème de l'échéance) que parce qu'ils sont au cœur de conflits d'intérêts. 


\section{CONCLUSION}

Une image peut être évoquée pour illustrer le traitement du risque par la comptabilité : à la page 9 de la bande-dessinée « Tintin au Tibet» (Hergé 1960), le capitaine Haddock, qui a une poussière dans l'œil, se concentre sur les marches de la passerelle qu'il escalade précipitamment pour rejoindre un avion... qui n'est pas relié à la passerelle ! «C'est heureux que j'y voie encore assez pour ne pas rater une marche ! »s'exclame-t-il. Concentré sur le risque, de court terme, de rater une marche, il oublie un risque pourtant majeur, celui de se tromper d'escalier, et la chute (au sens propre comme au sens figuré) brutale qui va en découler.

La comptabilité n'est-elle pas, à l'image du capitaine, focalisée sur le traitement des risques opérationnels quantifiables, et aveugle sur les risques majeurs? N'est-il pas évocateur, en parcourant les pages du rapport de gestion 2009 du groupe BP (BP 2009), publié quelques semaines avant l'accident du Golfe du Mexique de constater que la plaquette (et notamment sa couverture) est illustrée par de nombreuses photographies de plateformes dont la sécurité est citée (page 6) comme le premier élément de performance de l'entreprise ?

Si l'obligation de rendre des comptes existe, la comptabilité est incapable de traduire les risques les plus extrêmes, et notamment les risques de catastrophe nucléaire. Ce constat interroge le chercheur sur la nature des règles comptables : parce que l'information est un instrument dans la relation d'agence entre dirigeants et investisseurs dont les intérêts peuvent être contradictoires, la règle comptable sert, avant même de répondre à son objectif de fournir une image « fidèle » de la réalité, à protéger le comptable des influences des parties prenantes, et donc à assurer une neutralité procédurale qui peut nuire à l'objectif de représentation fidèle de la réalité de l'entreprise. 
L'étude du mécanisme de traitement comptable du risque le démontre: la démarche séquentielle exclut de fait les risques extrêmes malgré l'enjeu majeur qu'ils constituent pour la société. La conséquence directe de cette lacune concerne les premiers destinataires des comptes: les investisseurs. Ceux-ci ne sont pas informés des conséquences de risques extrêmes qui pèsent sur la santé (et donc sur le résultat distribuable) de l'entreprise.

La conséquence indirecte, mais pas moins importante, concerne la société dans son ensemble. La non prise en compte de coûts futurs potentiels minimise le coût de revient et donc le prix du $\mathrm{kWh}$ d'origine nucléaire. Cela a des conséquences sur les choix énergétiques des consommateurs et des États et transfère sur les générations futures les conséquences de ces choix.

Constituer une provision permet, en limitant le résultat distribuable, de constituer progressivement une réserve financière qui doit permettre de faire face à l'accident futur. Mais lorsque l'échéance est inconnue, la constitution de la provision est impossible. Une voie de sortie est cependant envisageable, en s'inspirant des approches développées pour encadrer les établissements de crédit et limiter les risques systémiques: obliger les entreprises à augmenter leurs capitaux propres, à respecter des ratios de couverture contraignants, et à posséder des actifs dédiés à la couverture des risques. Cette solution ne se traduit alors pas par une modification du résultat comptable, mais par une modification de la structure du patrimoine. Indirectement, le coût du $\mathrm{kWh}$ serait cependant affecté puisque l'accroissement des ratios de couverture oblige les entreprises à immobiliser d'importantes ressources financières, ce qui génère un coût d'opportunité.

En conclusion, l'analyse comptable du risque ne permet pas de traduire les risques extrêmes dans les états financiers, et les travaux des normalisateurs comptables, qui se traduisent dans l'évolution des normes IFRS, ne montrent pas d'évolution significative. Par contre, une intervention des États et une évolution de la réglementation s'inspirant des réflexions 
développées dans le cadre de l'analyse des risques pesant sur les établissements de crédit, pourraient tracer une voie de sortie salutaire.

\section{BIBLIOGRAPHIE}

Berkowitz, H. (2013). Les risques majeurs et l'action publique. Le Libellio d'AEGIS 9 (4): 13-20.

Bertel, E., Naudet, G. (2004). L'économie de l'énergie nucléaire. Paris: EDP Sciences.

BP. (2009). Annual review 2009 ; operating at the energy frontiers.

CNC. (2005). Avis relatif à la comptabilisation des coûts de démantèlement, d'enlèvement et de remise en état de site dans les comptes individuels, $n^{\circ} 2005-h$ du 6 décembre 2005 du comité d'urgence.

Colasse, B. (1999). Vingt ans de recherche comptable française : Continuité et renouveau. Comptabilité Contrôle - Audit: 23-34

Cour_des_comptes. (2005). Le démantèlement des installations nucléaires et la gestion des déchets radioactifs. Rapport au président de la république suivi des réponses des administrations et des organismes concernés. Paris. pp.292.

CRC. (2000). Règlement $n^{\circ}$ 2000-06 du 7 décembre 2000. Paris.

Dick, W., Eberhartinger, E. (2002). Les provisions pour risques et charges en france et en allemagne à la lumière des évolutions internationales récentes. Comptabilité - Contrôle - Audit 5 (nº spécial): 179-197.

Hergé (1960). Les aventures de tintin : Tintin au tibet. Bruxelles:

Hicks, J. (1939). Value and capital. Oxford, UK: University Press.

IRSN. (2005). Sarnet : Réseau d'excellence européen sur les accidents graves de réacteur nucléaire. Son déploiement et ses réalisations après une année de fonctionnement. Dossier de presse, Direction de la communication.

Moulin, J. (2003). Le risque, le salarié et l'entreprise : Contribution syndicale à la prévention des risques industriels et technologiques majeurs. Editions VO.

Schmitt, A., Spaeter, S. (2007). Risque nucléaire civil et responsabilité optimale de l'exploitant. Revue économique 58: 1331-1351. 